\title{
The molecular genetic basis of herbivory between butterflies and their host plants
}

\author{
Sumitha Nallu', Jason A. Hill2 , Kristine Don ${ }^{11}$, Carlos Sahagun', Wei Zhang ${ }^{1,6}$, Camille Meslin $\mathbb{1}^{3,7}$, \\ Emilie Snell-Rood4, Nathan L. Clark $\mathbb{1}^{3}{ }^{3}$, Nathan I. Morehouse ${ }^{5}$, Joy Bergelson', Christopher W. Wheat ${ }^{2}$ \\ and Marcus R. Kronforst ${ }^{1}{ }^{1 \star}$
}

Interactions between herbivorous insects and their host plants are a central component of terrestrial food webs and a critical topic in agriculture, where a substantial fraction of potential crop yield is lost annually to pests. Important insights into plant-insect interactions have come from research on specific plant defences and insect detoxification mechanisms. Yet, much remains unknown about the molecular mechanisms that mediate plant-insect interactions. Here we use multiple genome-wide approaches to map the molecular basis of herbivory from both plant and insect perspectives, focusing on butterflies and their larval host plants. Parallel genome-wide association studies in the cabbage white butterfly, Pieris rapae, and its host plant, Arabidopsis thaliana, pinpointed a small number of butterfly and plant genes that influenced herbivory. These genes, along with much of the genome, were regulated in a dynamic way over the time course of the feeding interaction. Comparative analyses, including diverse butterfly/plant systems, showed a variety of genome-wide responses to herbivory, as well as a core set of highly conserved genes in butterflies as well as their host plants. These results greatly expand our understanding of the genomic causes and evolutionary consequences of ecological interactions across two of nature's most diverse taxa, butterflies and flowering plants.

$\mathrm{B}$ utterflies and moths, and the host plants that their larvae feed on, comprise two of the largest groups of species on earth, the Lepidoptera and Angiosperms. The extreme diversity of these two groups has arisen from the co-evolutionary interactions between them, wherein evolution proceeded via reciprocal adaptations as each clade evolved in response to changes in the other ${ }^{1,2}$. A seminal study in this field $^{3}$ used the diffuse evolutionary relationships among butterflies and their host plants to formally introduce the concept of co-evolution. In the 50 years since that study ${ }^{3}$, research on the molecular basis of herbivory between butterflies and their host plants has revealed important insights into specific chemicals and defence pathways usedby plants, such as furanocoumarins, glucosinolates and cardenolides ${ }^{4}$. Research on butterflies, in turn, has revealed specific genes and gene families involved in host plant detoxification, such as cytochrome P450 enzymes ${ }^{5,6}$, the nitrile-specifier protein of pierid butterflies ${ }^{7}$ and the $\mathrm{Na}^{+} / \mathrm{K}^{+}$-ATPase of the monarch butterfly ${ }^{8}$. Yet, despite these important advances, our understanding of the molecular genetic basis of these co-evolutionary interactions remains limited. Genomic approaches, including genomewide association (GWA) studies and transcriptomics, provide a means to move beyond candidate genes and pathways to uncover the molecular determinants of this fundamental ecological interaction in an unbiased way ${ }^{9,10}$. Here we use these methods in a varieties of butterflies and their respective host plant to uncover the genetic basis of herbivory.

Results

Genome-wide associations with herbivory in the host plant Arabidopsis thaliana. First, we investigated the genetics of butterfly -host herbivory by mapping associated variants in parallel GWA studies focused on the flowering plant Arabidopisis thaliana and its natural insect herbivore, the cabbage white butterfly, Pieris rapae. For these experiments, we used either 96 natural accessions of A. thaliana (Supplementary Table 1) and a single lab strain of $P$. rapae (for the plant GWA studies), or the offspring of 96 field-caught females of $P$. rapae (Supplementary Table 2) and a single accession of $A$. thaliana (for the butterfly GWA studies). For both experiments, we measured herbivory as the amount of weight gained and the amount of leaf surface area eaten by second instar larvae over a period of $72 \mathrm{~h}$. A. thaliana GWA resulted in a total of 90 associated SNPs that were in linkage disequilibrium with 389 genes. Analysis of gene enrichment, using DAVID ${ }^{11}$, revealed plant defence as a prominent annotation cluster among these 389 genes (Supplementary Data 1). While plant defence was not significantly enriched after correcting for multiple comparisons, this category had an enrichment score of 1.43 , which is above the value (1.3) indicative of enrichment $^{11}$. A subset of 12 well-supported candidate genes contained three or more associated SNPs each (Fig. 1a). Eight of these genes were functionally validated using SALK T-DNA mutants, showing increased larval weight gain and/or increased plant material eaten in knockouts versus control (Fig. 1c and Supplementary Table 3). This validated gene set includes both well-known and novel defence genes. For instance, the cytochrome $\mathrm{P} 450$ gene CYP79B2 is involved in the conversion of tryptophan to indole-3-acetaldoxime, a precursor of indole glucosinolates and indole-3-acetic acid ${ }^{12}$. Indole glucosinolates are important secondary metabolites used for defence by

'Department of Ecology and Evolution, University of Chicago, Chicago, IL, USA. ${ }^{2}$ Department of Zoology, Stockholm University, Stockholm, Sweden. ${ }^{3}$ Department of Computational and Systems Biology, University of Pittsburgh, Pittsburgh, PA, USA. ${ }^{4}$ Department of Ecology, Evolution and Behavior, University of Minnesota, Saint Paul, MN, USA. ${ }^{5}$ Department of Biological Sciences, University of Cincinnati, Cincinnati, OH, USA. ${ }^{6}$ Present address: PekingTsinghua Center for Life Sciences, State Key Laboratory of Protein and Plant Gene Research, and School of Life Sciences, Peking University, Beijing, China. 7Present address: Institut National de la Recherche Agronomique (INRA), Institute of Ecology and Environmental Sciences of Paris (IEES-Paris), Versailles, France. *e-mail: mkronforst@uchicago.edu 

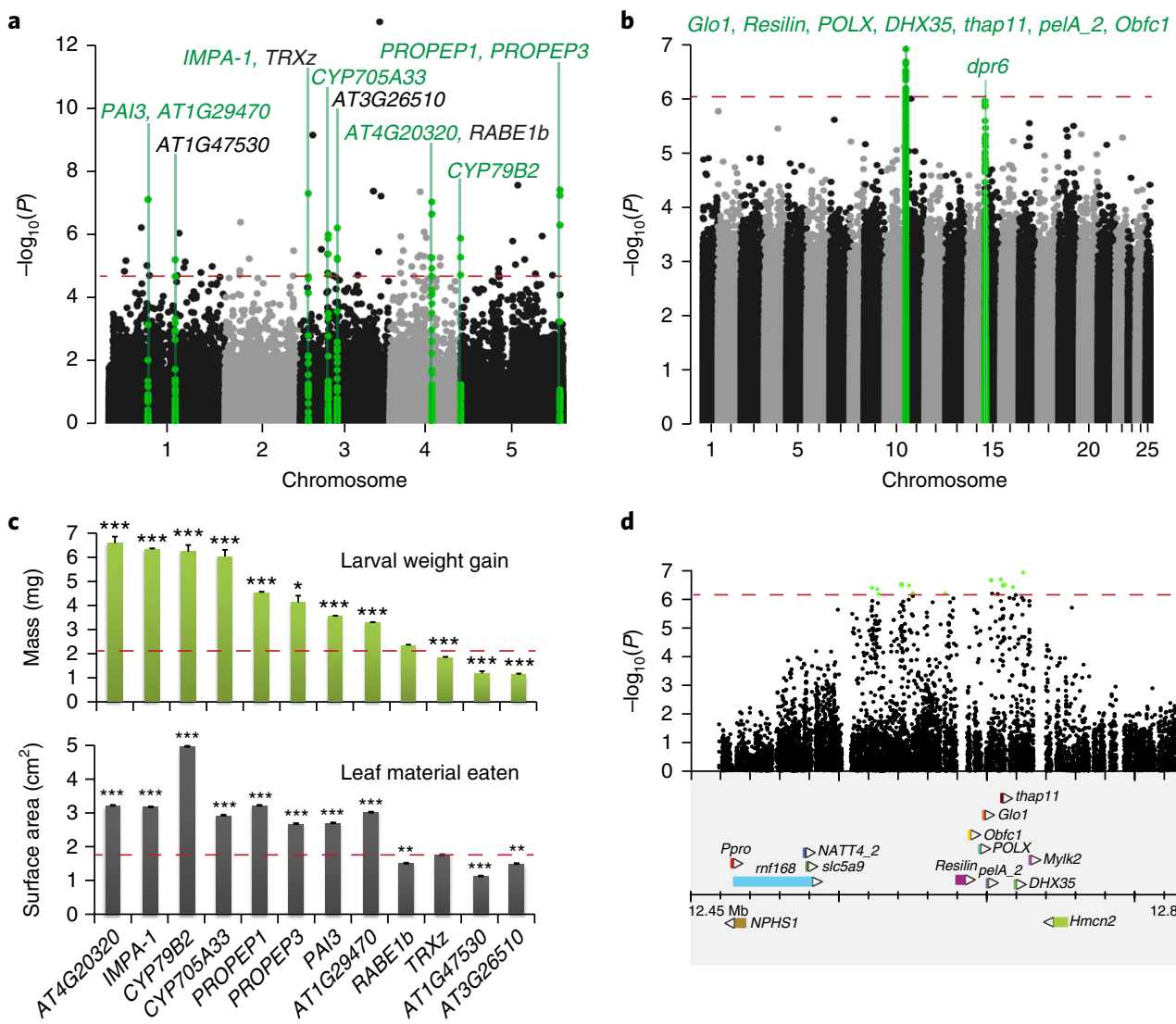

d

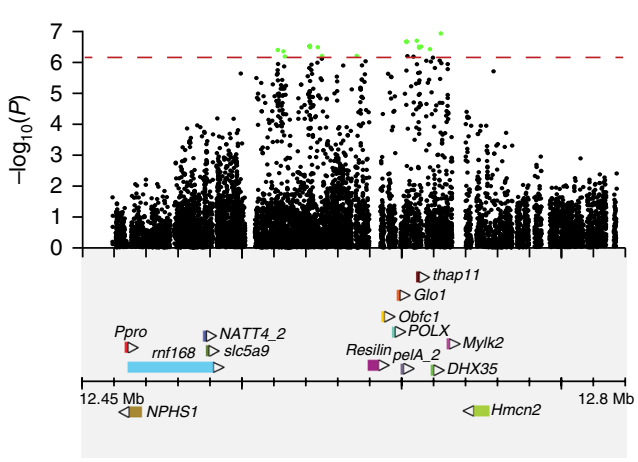

Fig. 1 | Butterfly and host plant herbivory candidate genes identified by parallel GWA studies. a, Manhattan plot with the associated SNPs and their corresponding P values from the herbivory GWA studies based on 96 accessions of $A$. thaliana. The 8 genes that were functionally validated among the primary 12 candidate genes are highlighted in green. $\mathbf{b}$, Manhattan plot with the associated SNPs and their corresponding $P$ values from the herbivory GWA studies based on 96 P. rapae samples. The genes contained in the two association peaks are listed. The significance threshold for each GWA study is indicated with a dashed red line. c, Average larval weight gain (top panel) and leaf surface area eaten (bottom panel) on the SALK T-DNA mutants for the 12 candidate genes from the Arabdiopsis GWA studies ( $\left.{ }^{\star} P<0.05,{ }^{\star \star} P<0.01,{ }^{\star \star \star} P<0.001\right)$. Plots show mean \pm s.e.m. and the corresponding mean for the wild-type plant (col-0) is represented as a dashed line across each histogram. Individual values, means and statistical tests are in Supplementary Table 3. d, Zoom-in on the chromosome 10 region associated with herbivory in the P. rapae genome. Significantly associated SNPs are indicated in green with a gene map below.

Arabidopsis, and other species in the plant family Brassicaceae, and they have been shown to deter herbivores and pathogens ${ }^{13,14}$. Insects that feed on Brassicaceae have evolved various physiological strategies against toxic effects of glucosinolates ${ }^{15}$. The larvae of $P$. rapae, for instance, redirect the hydrolysis pathway catalysed by myrosinase ${ }^{7}$, and instead of producing toxic isothiocyanates, hydrolysis is redirected towards the formation of nitriles by the butterfly's nitrilespecifier protein (NSP) ${ }^{16,17}$. Another functionally validated gene, phosphoribosylanthranilate isomerase 3 (PAI3), catalyses a step in the L-tryptophan synthesis pathway ${ }^{18}$ to produce the precursor of indole glucosionolates, although there is no direct evidence that it influences glucosinolates. The genes PROPEP1 and PROPEP3 belong to the AtPep (endogenous danger peptides) gene family. They are associated with activation of danger- or damage-associated molecular patterns (DAMPs) immunity in plants against both pathogen and herbivore attack ${ }^{19}$. The other four genes validated in our study do not have known roles in defence and include an uncharacterized cytochrome P450 (CYP705A33), Importin alpha (IMPA-1), a CTP synthase (AT4G20320), and an S-adenosyl-L-methionine-dependent methyltransferase (AT1G29470). The A. thaliana genes we found associated with herbivory did not overlap with those from a recent GWA study of methionine-derived glucosinolates in A. thaliana ${ }^{20}$. This is expected, perhaps, because these aliphatic glucosinolates do not seem to negatively impact $P$. rapae larvae ${ }^{21}$.
P. rapae genome sequencing. We began our butterfly GWA studies by assembling a high-quality reference genome sequence for $P$. rapae. For the reference genome, we combined next-generation DNA sequencing data from one PCR-free paired-end Illumina library, three mate-pair libraries ( $3 \mathrm{kbp}, 7 \mathrm{kbp}$ and $40 \mathrm{kbp}$ ), a Dovetail Chicago library, and information from the genome assembly of a closely related species, Pieris napi $i^{22}$, to generate an assembly of 323 Mbp, with a N50 of $11.5 \mathrm{Mbp}$, spanning the 25 P. rapae chromosomes $^{23}$ (Supplementary Figs. 1 and 2). Annotation was performed using a diverse set of RNA-seq data. For subsequent analyses, we removed unplaced scaffolds from the assembly that represented redundant haplotypes, yielding a chromosomal assembly of 272 Mbp. K-mer size estimation of the P. rapae genome was $289.8 \mathrm{Mbp}$. This haploid, chromosomal-level assembly of the butterfly genome provided an essential genomic foundation with which to explore the insect side of the plant-insect interaction.

Genome-wide associations with herbivory in the herbivore $\boldsymbol{P}$. rapae. For GWA, we performed our larval feeding trials with the offspring of wild-caught females and used whole-genome resequencing to genotype 96 unrelated larvae at genome-wide singlenucleotide polymorphisms (SNPs). P. rapae GWA revealed just two strongly associated regions in the genome (Fig. 1b), the largest of which encompassed a total of 16 significantly associated SNPs 
distributed across $98 \mathrm{kbp}$ on chromosome 10, spanning seven genes (Fig. 1d). One of these genes, Glyoxalase 1 (Glo1), a lactoylglutathione lyase, is a central enzyme in the glyoxalase pathway present in all organisms. Glyoxalase detoxifies cytosolic methyglyoxyl, a toxic by-product of metabolism, in a two-step process that utilizes glutathione. Glutathione itself is an important metabolite involved in multiple biological processes in plants and animals ${ }^{24}$. In addition to Glo1, the $98 \mathrm{kbp}$ associated region also contained the following genes: Resilin, a retrovirus-related Pol polyprotein POLX, the ATP-dependent RNA helicase DHX35, a THAP domain-containing protein, and two uncharacterized genes. A second associated region in the genome was prominent and close to the significance threshold so we explored it further. This region on chromosome 15 contained just one gene, Defective proboscis extension response 6 (dpr6) (Supplementary Fig. 3). The $d p r$ gene family is involved in the sensory physiology of adult Drosophila melanogaster, specifically response to gustatory cues ${ }^{25}$. Our results suggest this gene plays a role in butterfly larval growth, possibly by mediating gustatory response to host plant material or in the context of host plant detoxification. The GWA regions on chromosomes 10 and 15 exhibited evidence of balancing selection, including elevated nucleotide diversity $(\pi)$ and Tajima's Dvalues compared to the flanking 100 $\mathrm{kbp}$ upstream and downstream, as well as significant HKA tests for a number of genes within the chromosome 10 GWA interval (Supplementary Figs. 4 and 5).

Herbivory-induced differential gene expression experiments. To further interrogate the GWA genes, and explore genome-wide patterns of gene expression throughout the plant-insect interaction, we next used transcriptomics to measure differential gene expression in $P$. rapae and $A$. thaliana over the time course of their interaction. For these experiments, butterfly and plant were allowed to interact for a period of time, after which we harvested tissue from both organisms, as well as controls, for RNA-seq (Fig. 2a-f and Supplementary Table 4). For these experiments, the plant and butterfly genome sequences were used as references to analyse RNA-seq data. The genes identified in our butterfly and plant GWA experiments showed patterns of expression consistent with a role in herbivory (Fig. 3). In A. thaliana, 8 of 12 genes identified in the GWA study had the highest expression in leaves exposed to eggs and larvae and/or leaves with larvae only, including both cytochrome P450 genes and PROPEP3. PROPEP1 and PAI3 showed elevated expression in leaves with eggs only, followed by leaves with larvae only. The remaining two genes, IMPA-1 and RABE1b (one of the four genes that was not validated) had other expression patterns, showing elevated expression in mechanically wounded leaves and the leaf control, respectively (Fig. 3a). Statistical comparisons, using ANOVA and Tukey's HSD post-hoc tests, supported a number of these expression differences, notably for the two cytochrome P450 genes as well as PROPEP1 (Supplementary Table 5). In P. rapae, a number of the associated genes had the highest expression in larval treatments-the larva is the herbivorous life stage of the butterflyand were expressed at lower levels in eggs, pupae and adults. Notably, this pattern of expression was distinct from physically adjacent genes that were located outside the associated regions (Figs. 3b,c). Resilin expression was elevated specifically in larvae feeding on leaves, as opposed to those eating artificial diet, indicative of a direct role in the plant-insect interaction (Supplementary Table 5).

The genome-wide responses of Arabidopisis and Pieris, in terms of differential gene expression, were also striking. In particular, A. thaliana showed a massive response to butterfly oviposition with approximately $50 \%$ of the plant's genes changing expression, mostly upregulated, in response to a female butterfly laying eggs (Fig. 2g). The response of the plant to larval feeding, in contrast, was more modest. The egg is the first life stage of the herbivore that is in contact with the plant, and previous studies have shown that plants launch various defences against insects even before larval hatching $^{26}$. Egg-induced plant defence strategies include plant-mediated desiccation of eggs, egg dropping, egg crushing and egg killing ${ }^{26}$. We found that a total of 14,563 genes were differentially expressed in Arabidopsis leaves after P. rapae egg deposition (Fig. 2i). Genes belonging to defence and stress responses were enriched in all treatments (Supplementary Data 2)-in addition to genes involved in production of glucosinolates and glutathiones, these included protein kinases, proteolytic enzymes, oxidoreductases, peroxidases, NBS-LRR and defence signalling transcription factors that are known to be involved in release of reactive oxygen species, production of pathogenicity-related proteins, activation of systemic acquired resistance, cell wall modification and programmed cell death in pathogens ${ }^{27}$. These results mirror and greatly extend previous work with microarrays and a different species of Pieris, $P$. brassicae, showing an elevated response of $A$. thaliana to insect oviposition with induction of genes involved in the hypersensitivelike response and pathogenesis-related genes as well as callose and reactive oxygen species accumulation ${ }^{28-30}$.

Similar to the plant, $P$. rapae eggs exhibited an elevated response in comparison to the larval stages (Fig. $2 \mathrm{~h}$ ). Of all the genes differentially expressed between eggs oviposited on leaves and wax paper, approximately $50 \%$ of them were uncharacterized. Among the genes with predicted functions, genes responding to stress, oxidoreductases, and proteolytic enzymes were abundant (Supplementary data 3). In the next stage of their interaction (larvae feeding on the plant), we analysed gene expression patterns in larvae feeding on plants exposed to eggs as well as those feeding on plants not previously exposed to eggs. We found that larvae feeding on plants exposed to eggs had a smaller number of differentially expressed genes compared to larvae feeding on plants that were not exposed to eggs (Fig. $2 \mathrm{j}$ ). This mirrored gene expression patterns in the plant (Fig. 2i), in which the added effect of herbivory after oviposition was less than the effect of herbivory alone. There were 120 differentially expressed larval genes in common between the two treatments, and among the genes with putative functions, genes involved in defence, stress response and proteolysis were overrepresented (Supplementary Data 4). Interestingly, glutathione $s$-transferases (GSTs) frequently show elevated expression in the larvae of Lepidopter ${ }^{31-33}$ and other insects ${ }^{34}$, but we did not detect differential expression of GSTs in P. rapae.

Comparative transcriptomics across butterflies and plants. The strong response of $A$. thaliana to butterfly eggs was a particularly striking result. To determine if this was a general property of butterfly-plant interactions, we expanded our analysis of gene expression to three additional plant/insect systems: Medicago sativa/Colias eurytheme, Citrofortunella microcarpa/Papilio polytes and Passiflora oerstedii/Heliconius cydno (Fig. 4a). For this comparative analysis, we generated de novo transcriptomes for all organisms to use as references to analyse patterns of differential gene expression using RNA-seq data. We also reanalysed our A. thaliana/P. rapae data using de novo reference transcriptomes, as opposed to the genome sequences, for consistency. Interestingly, we found substantial variation in the responses of both plants and butterflies, and no plant showed the same, elevated response to oviposition that we saw in A. thaliana (Fig. 4b,c). To investigate which, if any, components of the defence network were conserved among plants or among butterflies, we extracted and compared common differentially expressed genes across the four systems. Among plants, common gene families that were differentially expressed in leaves after oviposition were protein kinases, proteases, heat shock proteins, esterases, MYB transcription factors and NAC transcription factors (Supplementary Data 5), all of which have previously been implicated in defence against pathogens ${ }^{35,36}$. During larval feeding, leaves of the four plant species shared protein kinases, proteases, heat shock proteins, esterases, MYB transcription factors, NAC transcription factors and, 


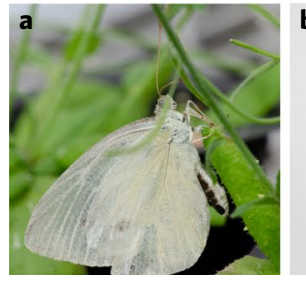

$\mathbf{g}$

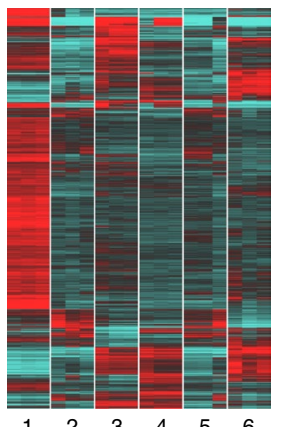

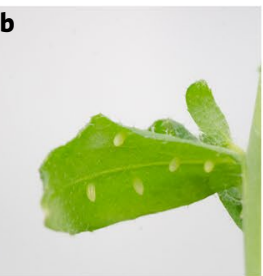

h

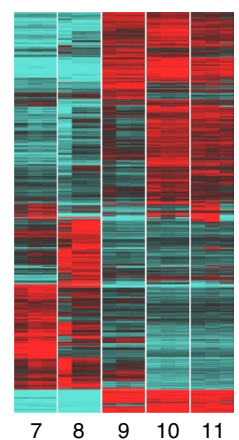

i
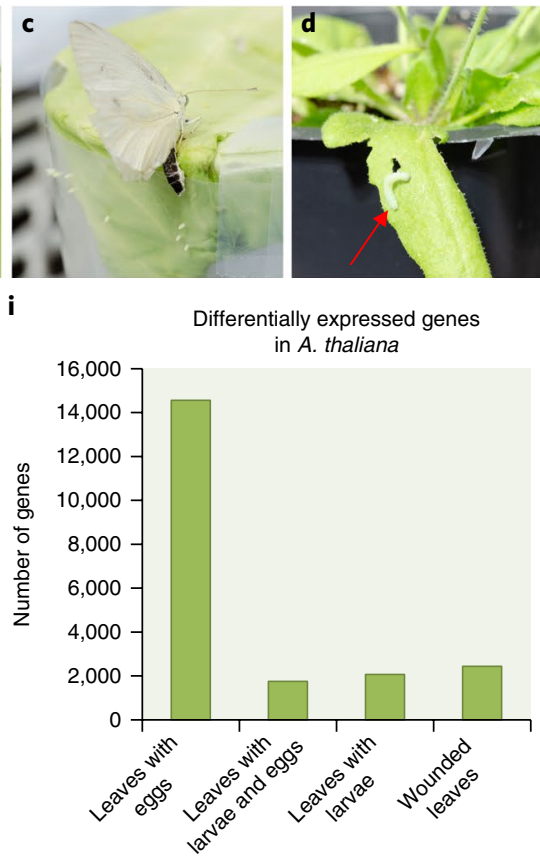
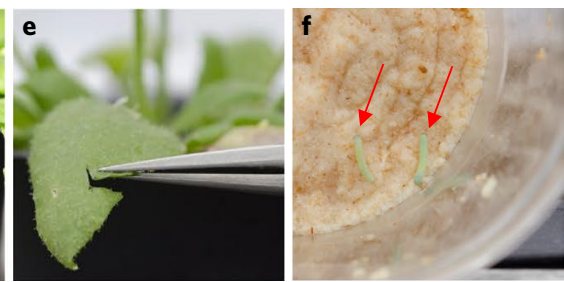

j

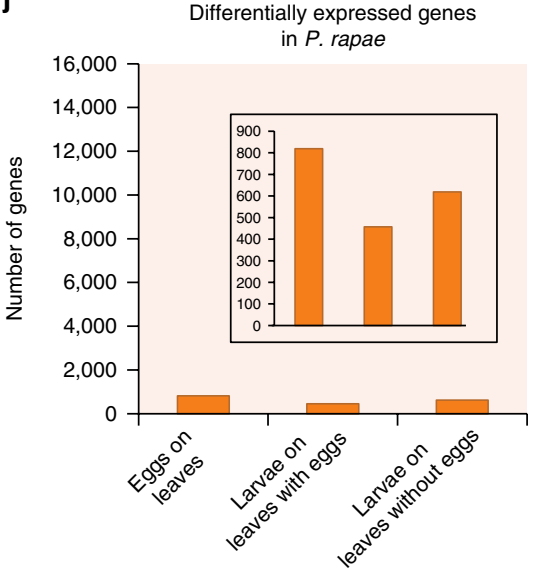

Fig. 2 | Genome-wide responses to herbivory in $\boldsymbol{A}$. thaliana and $\boldsymbol{P}$. rapae over the time course of their interaction. a-f, Samples collected from various stages of interaction: eggs laid on the leaves (a,b), eggs laid on the wax paper (c), larva feeding on a leaf (d), mechanical wounding of the leaf (e) and larvae feeding on artificial diet (f). $\mathbf{g}, \mathbf{h}$, Heat maps of differentially expressed genes in $A$. thaliana ( $\mathbf{g})$ and $P$. rapae (h). The colour scale ranges from $\leq-1.5 \log$ fold change (blue) to $\geq 1.5 \mathrm{log}$ fold change (red). Individual treatments correspond to: (1) leaves with eggs $72 \mathrm{~h}$ after oviposition; (2) leaves with no eggs, control for $72 \mathrm{~h}$ oviposition treatment; (3) leaves after $24 \mathrm{~h}$ larval (48 h old) feeding; (4) leaves with eggs after $24 \mathrm{~h}$ larval (48 h old) feeding; (5) leaves with no eggs and larvae, control for larval feeding and wounding; (6) leaves $24 \mathrm{~h}$ after wounding; (7) eggs $72 \mathrm{~h}$ after oviposition on wax paper; (8) eggs $72 \mathrm{~h}$ after oviposition on leaves; (9) $48 \mathrm{~h}$ old larvae after $24 \mathrm{~h}$ feeding on artificial diet; (10) $48 \mathrm{~h}$ old larvae after $24 \mathrm{~h}$ feeding on leaves with eggs; (11) $48 \mathrm{~h}$ old larvae after $24 \mathrm{~h}$ feeding on leaves. $\mathbf{i}$, The number of differentially expressed $A$. thaliana genes across treatments. $\mathbf{j}$, The number of differentially expressed $P$. rapae genes across treatments. The inset shows the same results plotted with a reduced $y$-axis scale. Details of treatment and control conditions are in Supplementary Table 4.

in addition, GSTs, cytochrome P450s, xyloglucan endotransglycosylases, Dof zinc finger proteins and WRKY transcription factors (Supplementary Data 6). Based on protein alignments, we identified a small set of 11 true orthologues among the differentially expressed genes, which were shared across all plant species. Leaves after oviposition did not have any shared orthologues but the larval feeding treatments had 4 or 5 orthologues each (Supplementary Data 7), the majority of which have putative roles in defence.

Among butterfly species, the common gene families that were differentially expressed in eggs $72 \mathrm{~h}$ following oviposition were proteases, heat shock proteins, esterases, chemosensory proteins and cuticular proteins (Supplementary Data 8), all of which have previously been implicated in detoxification pathways ${ }^{37,38}$. In larvae feeding on plants, the genes differentially expressed belonged to gene families such as chitinases, proteases, cuticular proteins, lipases and Osiris (Supplementary Data 9). There was a small set of 17 differentially expressed genes that were true orthologues shared among all butterfly species (Supplementary Data 10). Among this set of conserved genes, Osiris 9 (Osi9) stood out because it was upregulated in all four butterfly species, specifically in larvae feeding on leaves with previous exposure to eggs. Osi9 is a transmembrane protein that is a member of the 24 gene Osiris family that is unique to insects ${ }^{39}$. The function of Osiris genes has been mysterious ${ }^{39,40}$ but the Osiris gene cluster was recently associated with detoxification of the Morinda citrifolia host plant in both Drosophila sechellia ${ }^{41,42}$ and Drosophila yakuba ${ }^{43}$. Previous work has shown that Lepidoptera appear to have multiple copies of $\mathrm{Osi}^{39}$, and we detected multiple paralogues of Osi9 in the P. rapae genome and across our assembled transcriptomes (Supplementary Fig. 6). However, the copy of Osi9 that was upregu- lated in larvae of all four butterfly species was the same, Osiris $9 E$ (Supplementary Fig. 3). To further explore the potential role of Osi9E in butterflies, we surveyed spatial and temporal patterns its expression in the four butterfly species and found elevated expression during larval stages, as opposed to pupae and adult, and expression specifically in the larval gut (Supplementary Figs. 7 and 8 and Supplementary Tables 6 and 7). These results suggest Osi9E expression is upregulated in response to contact with host plant tissue.

\section{Discussion}

Butterflies and their larval host plants provide a historically significant example of co-evolution, and research has explored the molecular genetic basis of this ubiquitous ecological interaction ${ }^{5-8,16,17,32,33}$. Here we used two genome-wide approaches-GWA studies and transcriptomics - to characterize the genetic basis of herbivory in butterflies and plants simultaneously. Our GWA studies uncovered a relatively small number of well-supported herbivory genes in both A. thaliana and $P$. rapae. The A. thaliana GWA studies yielded 12 genes that contained 3 or more associated variants, 8 of which we were able to validate with knockout lines, and this gene set contained both established and novel plant defence genes. P. rapae GWA studies revealed just one strongly associated region of the genome that contained only 7 genes. One of these genes, Glyoxalase 1 stands out as a particularly good candidate gene because it uses glutathione to detoxify methylglyoxal, a toxic by-product of cellular metabolism. Glutathione is a defensive compound in plants: glutathione concentrations increase in plants during oxidative stress ${ }^{4-47}$ and has been shown to be involved in defence against pathogens ${ }^{48}$ and insect feeding ${ }^{49}$. Furthermore, glutathione levels are known to vary among 


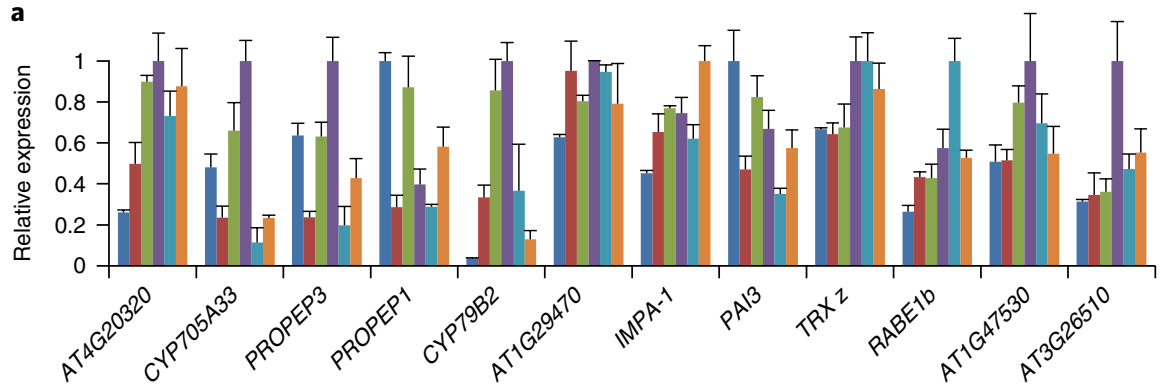

\author{
Leaves with eggs \\ - Leaf control for eggs \\ Leaves with larvae \\ - Leaves with larvae and eggs \\ - Leaf contol for larvae \\ Wounded leaves
}
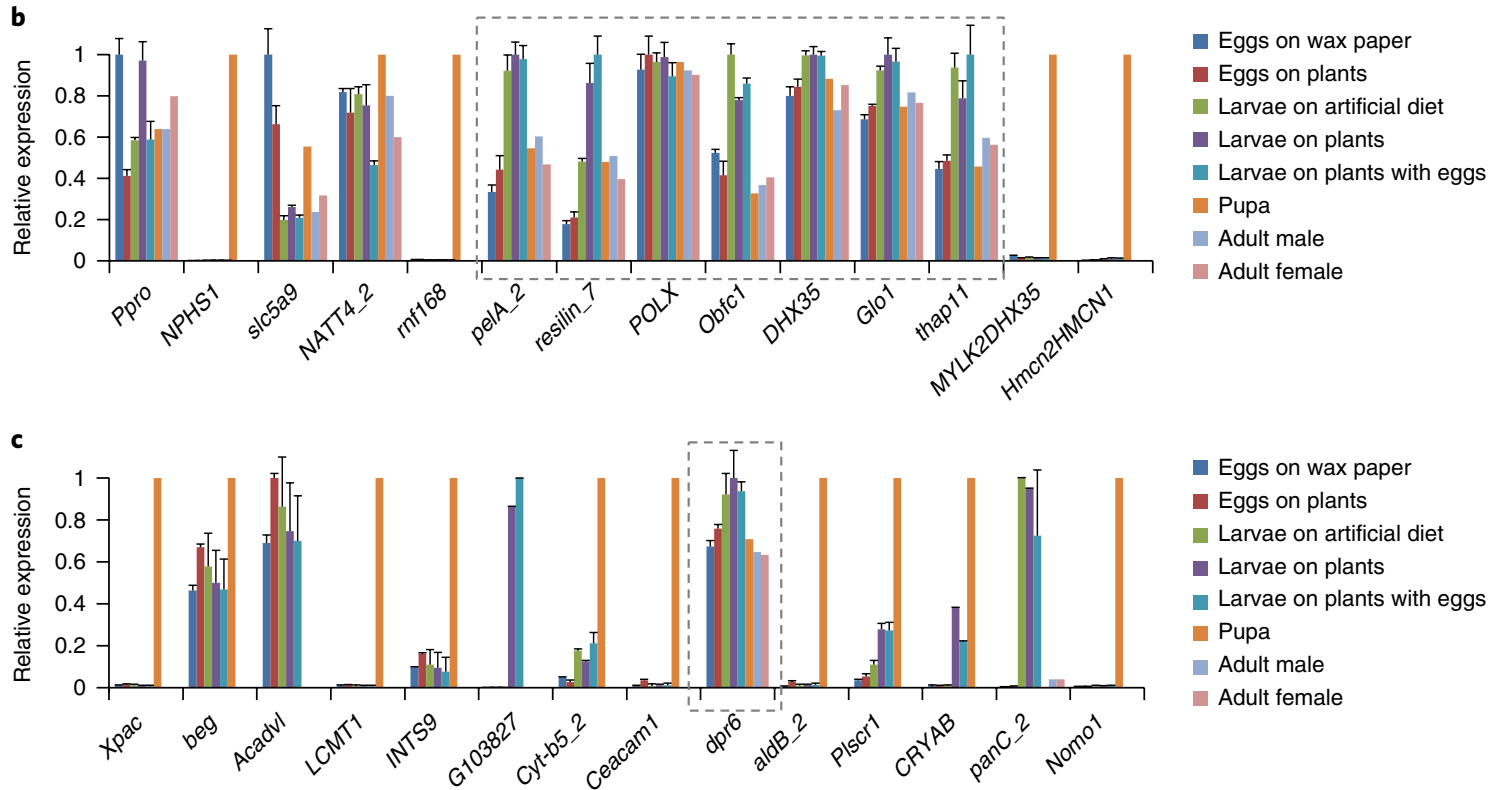

Fig. 3 | Temporal expression patterns of herbivory candidate genes in $\boldsymbol{A}$. thaliana and $\boldsymbol{P}$. rapae. a, Expression patterns from various RNA-seq treatments of the 12 A. thaliana candidate genes identified in the herbivory GWA studies. $\mathbf{b}$, Expression patterns from various RNA-seq treatments of the 7 P. rapae genes contained in the associated region on chromosome 10 (boxed), identified in the $P$. rapae herbivory GWA studies, as well as genes from the adjoining $100 \mathrm{~kb}$ regions upstream and downstream. $\mathbf{c}$, Expression patterns from the various RNA-seq treatments of the dpr6 gene identified in the P. rapae herbivory GWA studies, as well as genes from the adjoining $100 \mathrm{~kb}$ regions upstream and downstream. Plots show mean \pm s.e.m of gene expression values, fragments per kilobase of transcript per million fragments mapped (FPKM), scaled to the highest value treatment for each gene. Individual values, means and statistical tests are in Supplementary Table 5.

Arabidopsis accessions ${ }^{50}$ and we found natural sequence variation in the GST genes in our mapping panel (Supplementary Data 11). It is intriguing to consider the possibility that $P$. rapae may somehow be using the glyoxalase pathway to detoxify host plant glutathione. A second $P$. rapae GWA peak, while not statistically significant, was notable and contained just a single gene, dpr6. Defective proboscis extension response $(\mathrm{dpr})$ genes are a subclass of immunoglobulindomain-containing proteins that interact with Dpr-interacting proteins (DIPs) to specify synaptic connections ${ }^{51}$. Functionally, $d p r 6$ is an intriguing herbivory candidate gene because the $d p r$ gene family is known to influence the response of Drosophila melanogaster to gustatory cues ${ }^{25}$. The combined $P$. rapae GWA results suggest that we may have identified genes related to host plant detoxification and/or larval metabolism and growth.

Previous research on P. rapae and related butterflies has identified the NSP as a critical component in host plant detoxification ${ }^{7}$. The origin of NSP appears to be the key innovation that allowed the ancestor of the butterfly subfamily Pierinae to colonize and detoxify host plants in the order Brassicales, all of which produce glucosinolates (mustard oils) ${ }^{16,17}$. Before to our experiments, we hypothesized that genetic variation at NSP may also influence detoxification capacity in contemporary populations and that we may see associations with
NSP in our butterfly GWA study. This was not the case, but it is possible that a butterfly GWA study that incorporated other phenotypes, such as larval survival or development time, might detect associations with NSP. Furthermore, NSP was not differentially expressed in our transcriptomic experiments with $P$. rapae, although it was expressed in all larvae. In contrast, glucosinolate pathway genes did emerge in the Arabidopsis GWA studies and many were differentially expressed in our transcriptomic studies, suggesting that glucosinolate defence remains an active front in this co-evolutionary arms race, at least on the plant side of the interaction.

Expanding our analysis to four diverse butterfly/plant systems, we found that over evolutionary time, the molecular dynamics of the plant-insect interaction change dramatically. The number, timing and identity of genes expressed in plants and butterflies throughout the plant-insect interaction differed considerably across the four systems we studied. This finding highlights that the specific genetic underpinnings of herbivore-plant dynamics derived from a model system such as Arabidopsis may not always be generalizable to other systems. However, such a result is also expected because the process of co-evolution should drive each system along a very different evolutionary trajectory, especially over the long times scales that separate the butterfly and plant species we are studying. What is 


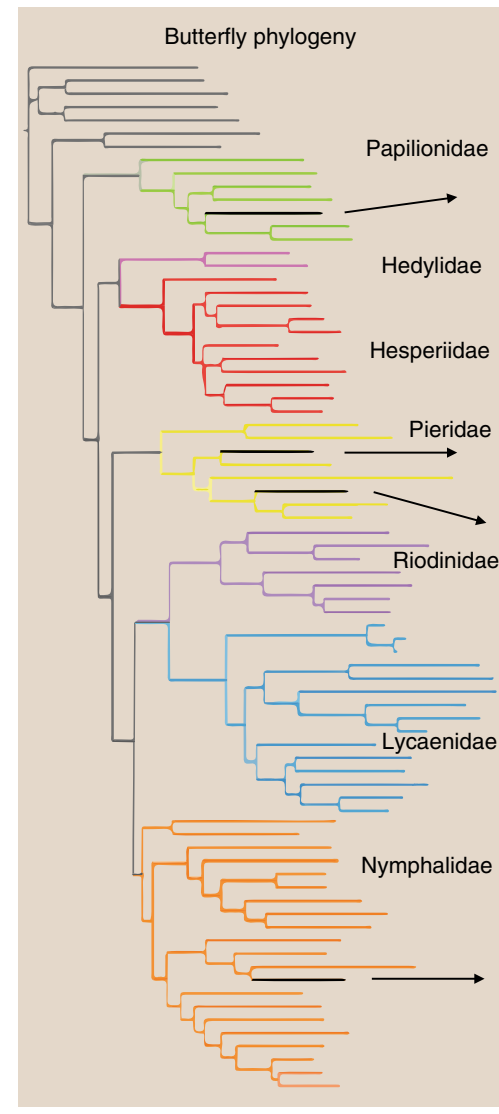

b

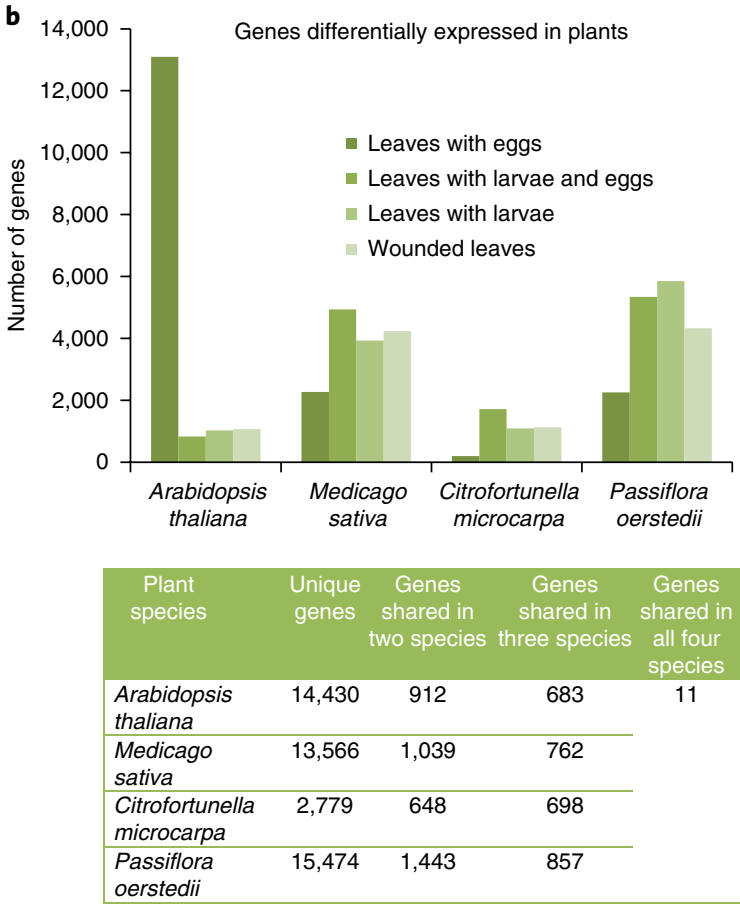

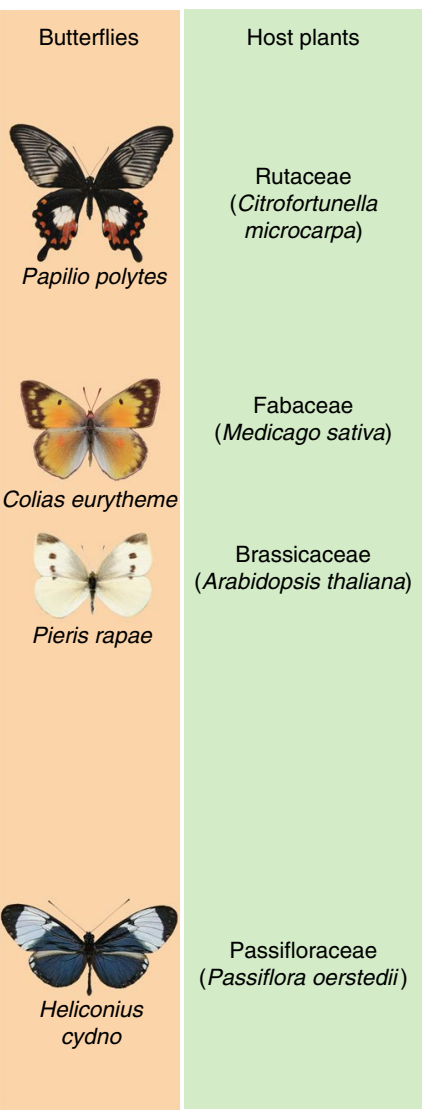

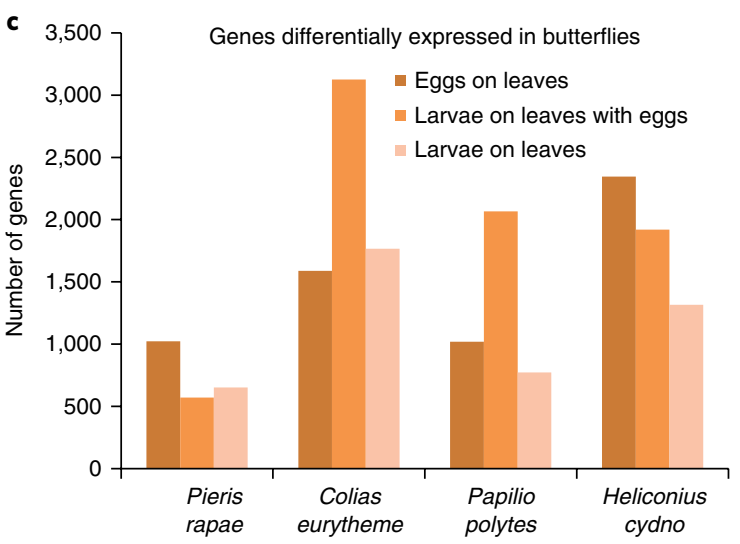

\begin{tabular}{|c|c|c|c|c|}
\hline $\begin{array}{l}\text { Butterfly } \\
\text { species }\end{array}$ & $\begin{array}{l}\text { Unique } \\
\text { genes }\end{array}$ & $\begin{array}{c}\text { Genes } \\
\text { shared in } \\
\text { two species }\end{array}$ & $\begin{array}{c}\text { Genes } \\
\text { shared in } \\
\text { three species }\end{array}$ & $\begin{array}{l}\text { Genes } \\
\text { shared in } \\
\text { all four } \\
\text { species }\end{array}$ \\
\hline $\begin{array}{l}\text { Pieris } \\
\text { rapae }\end{array}$ & 1,389 & 417 & 423 & 18 \\
\hline $\begin{array}{l}\text { Colias } \\
\text { eurytheme }\end{array}$ & 5,263 & 706 & 492 & \\
\hline $\begin{array}{l}\text { Papilio } \\
\text { polytes }\end{array}$ & 2,981 & 483 & 376 & \\
\hline $\begin{array}{l}\text { Heliconius } \\
\text { cydno }\end{array}$ & 4,668 & 472 & 423 & \\
\hline
\end{tabular}

Fig. 4 | Comparative transcriptomics of herbivory across four diverse butterflies and their host plants. a, Butterfly phylogeny (adapted from ref. ${ }^{92}$ ) displaying the four butterfly species included in the comparative transcriptomics analysis and their respective host plants. $\mathbf{b}, \mathbf{c}$, The number of differentially expressed genes across treatments for each host plant (b) or butterfly (c) species (top), and counts of differentially expressed genes that are unique or shared across species (bottom).

surprising, then, is that we did identify a core set of orthologous genes that were differentially expressed in response to herbivory in all butterfly-plant systems. A total of 11 orthologues were differentially expressed in all plant species and 17 in butterflies, but this summary belies further complexity as a number of these genes, while all differentially expressed, were regulated in opposite 
directions among systems, being upregulated in one species and downregulated in another. One gene that stood out among the conserved orthologues in butterflies was Osiris $9 E$, both because it was consistently expressed at high levels in larvae of all four butterfly species and because Osiris genes were recently implicated in host plant detoxification in Drosophila ${ }^{41-43}$. Furthermore, Osiris 9 and a number of other Osiris genes have been shown to be differentially expressed in the larvae of the fly Scaptomyza flava in response to feeding on glucosinolates ${ }^{52}$. These results suggest that Osiris genes are ancient players in insect-plant interactions.

As a whole, our results provide a comprehensive portrait of the molecular genetic dynamics mediating insect-host interactions. Importantly, we are able to not only corroborate previous findings related to the identity of particular defence genes and pathways in Arabidopsis, but also expand this to other plants as well as the insect herbivores. The results from Pieris and other butterflies pinpoint specific genes that appear important for herbivory, and these results further hint at even deeper evolutionary ties among herbivorous insects as a whole. Important next steps will be to functionally characterize these genes and identify the molecular and cellular mechanisms by which they impact herbivory. A complete understanding of the molecular genetic basis of the pervasive, antagonistic relationship between caterpillars and their host plants promises to inform our understanding of ecology, evolution and human agriculture.

\section{Methods}

Genome assembly and annotation. Genomic DNA was isolated from single, seventh-generation inbred female $P$. rapae pupae using ethanol precipitation ${ }^{53}$. This inbred line of $P$. rapae was established using a singly mated wild female caught in August 2013 near Rochester, Pennsylvania, USA ( $+40^{\circ} 44^{\prime} 45^{\prime \prime},-80^{\circ} 9^{\prime}$ $\left.45^{\prime \prime}\right)$. Three Illumina libraries were prepared, one PCR-free DNA library (180 bp) and two mate-pair libraries $(3 \mathrm{~kb}$ and $7 \mathrm{~kb}$ ). The $180 \mathrm{bp}$ library was sequenced in two lanes and the two mate-pair libraries were sequenced in one lane each on Illumina HiSeq High Output mode, PE $100 \mathrm{bp}$. Genomic DNA was isolated as described above from another inbred $P$. rapae pupae, a sibling of the first sample described above, and a $40 \mathrm{~kb}$ mate pair library was constructed and sequenced by using a Lucigen NxSeq $40 \mathrm{kbp}$ Mate-Pair Cloning Kit. For the final scaffolding step variable insert size libraries of 100-100,000 bp, using DNA from a third sibling, were generated using the Chicago and HiRise methods ${ }^{54}$ and these were sequenced by Centrillion Biosciences Inc. (Palo Alto, CA, USA), Illumina HiSeq High Output mode, PE 100bp.

Genome size was estimated at 289.8 Mbp from unique k-mer distribution of the raw data using Jellyfish (version 2.1 .3 ) ${ }^{55}$ and a custom R script. For the $3 \mathrm{~kb}$ and $7 \mathrm{~kb}$ libraries, Nextclip (version 0.8$)^{56}$ was used to look for the absence of linker sequence in either read in a pair and discard those reads as potential contamination of non-mate pair sequence. All read sets were then quality filtered, the ends trimmed of adapters and low quality bases, and screened of common contaminants using bbduk (version 34.94, https://github.com/BioInfoTools/ BBMap/blob/master/sh/bbduk.sh). For contig generation and scaffolding, the $180 \mathrm{bp}, 3 \mathrm{~kb}$, and $7 \mathrm{~kb}$ reads were assembled using AllpathsLG (version 50960) ( $^{57}$. The best assembly was obtained by using a random subset of 56 million reads (33\%) from the initial $3 \mathrm{~kb}$ and $7 \mathrm{~kb}$ libraries with the full set of 162 million reads from the $180 \mathrm{bp}$ library, more input data resulted in reduced performance and quality of assembly. AllpathsLG was run with haploidify $=$ true option to compensate for the high degree of heterozygosity present in the $P$. rapae data. The assembly was composed of 318 million bases contained in 15,799 scaffolds with a N50 of 54,044bp. Complete conserved single copy orthologue content was assessed at $87 \%$ by CEGMA (version 2.5$)^{58}$. A second scaffolding step using SSPACE v2 ${ }^{59}$ and the $3 \mathrm{~kb}, 7 \mathrm{~kb}$, and $40 \mathrm{~kb}$ libraries together brought up the assembly size and the $\mathrm{N} 50$ to $143,392 \mathrm{bp}$. A final scaffolding step was undertaken by Dovetail genomics using the custom library and the HiRise scaffolding pipeline ${ }^{54}$, which improved the N50 to $3,706,409 \mathrm{bp}$.

The HiRise assembly was assessed with BUSCO (version 3.0.2) ${ }^{60}$ and found to contain $95.9 \%$ of complete single copy orthologues in the insecta protein set and $9.5 \%$ gene duplication (Supplementary Table 8 ). This was reduced to $5.5 \%$ duplication in the chromosomal assembly due to the selection of a single duplicated region for inclusion in the assembly from those that aligned to Pieris napi (Supplementary Table 8 ). The unplaced scaffolds of the full assembly account for $4.1 \%$ of the duplicated and $8 \%$ of the missing orthologues in the chromosome only assembly. Scaffolds that aligned to $P$. napi were left unplaced if the fell completely within a larger aligned scaffold. If there was a partial overlap, the smaller scaffold was broken and the unique fragment was placed adjacent to the larger scaffold (details in Supplementary Material finalPrapae.agp). Chromosomal relationships of scaffolds were inferred from alignment using LAST (version 714) ${ }^{61}$ to the chromosomal structure of a closely related species $P$. napi $i^{22}$ (Supplementary Fig. 1), and validated with scaffold junction spanning mate pair and syntenic blocks (Supplementary Fig. 2). The final P. rapae assembly contained $323,179,347$ bp in 25 chromosomes and 2,747 unplaced scaffolds and a N50 of $11,535,178$ bp. After excluding the unplaced scaffolds, a 272,024,799bp haploid, chromosomal assembly was generated that served as the reference $P$. rapae genome for further analyses.

Our genome sequencing project was independent of another recently published $P$. rapae genome sequence ${ }^{62}$. The previously published genome sequence of $P$. rapae was $246 \mathrm{Mbp}$, compared to our chromosomal assembly of $272 \mathrm{Mbp}$, which prompted us to investigate the differences between the genomes. We found that $95 \%$ of the previously published assembly was contained in our HiRise scaffolds, covering an average of $56 \%$ of the HiRise scaffolds. Only 594 HiRise scaffolds had no coverage at all from the previous assembly, accounting for $5 \mathrm{Mbp}$.

The HiRise assembly was annotated using RNA-seq derived from $P$. rapae that were sampled in September 2013 from a continuous, inbred lab colony established in October 2012 from 4 females collected in Rochester, Pennsylvania. All pooled individuals were full siblings. Tissue specific samples included 3 individuals for the male reproductive tract, 5 individuals for the female reproductive tract, and 80 salivary glands from 40 individuals. Subsequent genome annotation involved the following pipeline: (a) collection of reference proteins from Uniprot database ${ }^{63}$ and assembly of high-confidence transcript sequences from previously published RNA-seq data ${ }^{64}$ using tophat2 (version 2.0.9) ${ }^{65,66}$ and the cufflinks package (version 2.2.1) ${ }^{67}$; (b) modelling of repeat sequences to mask the genome using RepeatMasker package (version 4.0.3, Smit, AFA, Hubley, R \& Green, P. RepeatMasker Open-3.0.1996-2010, http://www.repeatmasker.org) and RepeatModeler package (version 1.0.8, Smit, AFA, Hubley, R. RepeatModeler Open-1.0.2008-2015, http://www.repeatmasker.org); (c) evidence-based gene build to generate training models for ab initio gene finders using the Maker package (version 2.31-6) ; $^{6}$; (d) manual curation of gene models and training of the Augustus gene finder (version 2.7) ${ }^{69}$; (e) re-annotation of the evidence-based annotation using ab initio predictions; and finally (f) functional annotation of the refined gene build using Blast matches against Uniprot/Swissprot and results from InterproScan, condensed and reconciled using ANNotation Information Extractor $\left(\right.$ Annie $^{70}$ ). This process produced a gene build with a final count of 15,047 genes and 35,824 mRNAs.

Sample collection and data analysis for GWA studies. Host plant GWA studies. The growth chamber conditions for growing $P$. rapae and A. thaliana were $23^{\circ} \mathrm{C}$ day $/ 21^{\circ} \mathrm{C}$ night and $60 \%$ relative humidity on a $16 \mathrm{~h}$ photoperiod. Three replicates of each of the 96 accessions of $A$. thaliana that are listed in Supplementary Table 1 were grown until they were almost ready to bolt. After taking a picture of each plant, two 5-day-old, lab-grown P. rapae larvae were weighed and placed on each plant and then the plant was enclosed in a plastic sleeve bag. After $72 \mathrm{~h}$, the larvae were weighed and a new picture of the plant was taken to record plant surface area eaten by the larvae. The weight gained by the larvae and the total surface area eaten were calculated and used as the phenotype data for GWA studies (Supplementary data 12). The SNP information for the 96 accessions from the $250 \mathrm{~K} \mathrm{SNP} \mathrm{data}{ }^{71}$ and the phenotype data were fit using a multivariate linear mixed model in GEMMA (version 0.94.1) for association studies ${ }^{72}$. The pipeline involves converting the SNP file into a PLINK binary PED file and generating a relatedness matrix file using default parameters. The average initial weight of the larvae was used as a covariate for the analysis. For the functional validation of the candidate genes, knock out T-DNA mutants from SALK and wild type plants were grown and assayed as described above.

Herbivore GWA studies. 96 P. rapae females were collected from various locations across the US Midwest during June and July 2014 and raised in the lab green house. These butterflies included 57 from around the University of Chicago campus, 19 from Schaumburg, IL, and 16 from North Dakota, 3 from downtown Chicago, and 1 from Carolina Biological Supply. The growth chamber conditions for growing P. rapae and $A$. thaliana were $23^{\circ} \mathrm{C}$ day $/ 21^{\circ} \mathrm{C}$ night and $65 \%$ relative humidity on a $16 \mathrm{~h}$ photoperiod. Eggs were collected from each female and two 5-day-old larvae from each family were weighed and placed together on a Col-0 A. thaliana plant; this was performed in triplicate. Leaf area eaten and weight gain phenotypes were assayed as described for the Arabidopsis GWA studies (Supplementary data 13).

For genotyping, we selected one random GWA study larva from each family (a total of 96 larvae), sequenced the genome of each sample to a mean coverage of $13.8 \times$ (Supplementary Table 2), and analysed the data using the P. rapae genome assembly. Medium coverage sequencing depth $(5-20 \times)$ is considered the most cost-effective choice for sample sizes close to 100 individuals $^{73}$ and variation in coverage did not introduce spurious population structure. The PCA plot was generated using the '-pca' option in PLINK (version 2.0) ${ }^{74}$ (Supplementary Fig. 9) and the admixture plot was generated using the default options of ADMIXTURE (version 1.3) $)^{75}$ (Supplementary Fig. 10).

Genomic DNA was extracted from skin tissue of the larvae using the VDRC Drosophila genomic extraction (https://stockcenter.vdrc.at/images/downloads/ GoodQualityGenomicDNA.pdf) and sequencing libraries were prepared using KAPA Hyper Prep Kits (KR0961 v1.14). Barcoded libraries were pooled and 
sequenced on an Illumina HiSeq 2500 to generate paired-end $100 \mathrm{bp}$ data. The average QPhred score of the 96 samples was 36.2 and no sample had less than 35 (a Phred score of 20 corresponds to a 1\% error rate). Raw reads were further passed through additional quality control software Trimmotatic (version 0.36$)^{76}$, where all bases in the reads that were below a quality score of 15 were trimmed using a sliding window of $4 \mathrm{bp}$ and all reads less than $36 \mathrm{bp}$ in length were discarded. Trimmed reads were aligned to the $P$. rapae reference genome using bowtie $2^{77}$, using a '-very-sensitive-local' option, and the aligned SAM files were prepared for calling SNPs using PICARD tools (version 1.141, http://broadinstitute.github.io/ picard). Three PICARD tools were used with default parameters in the following order: SortSam, AddOrReplaceReadGroups, and MarkDuplicates. GATK (version 3.4 $)^{78,79}$ was then used to call SNPs. During this process, we used BaseRecalibrator to readjust and correct any errors in the quality scores assigned by the sequencing machine, RealignerTargetCreator to locally realign reads reducing the number of mismatches, and IndelRealigner. Finally, UnifiedGenotyper was used to call SNPs jointly for all 96 samples with a minimum phred-scaled confidence threshold of 50. The accuracy of SNP calling was increased by providing all the samples jointly because the variant caller estimates posterior probability likelihoods at each site using the information from all samples and then proceeds to assign SNP genotypes. We used -dcov (downsampling of coverage) of 150 for uniformity among samples.

The SNP information for the 96 larvae (a total of 18,603,675 SNPs) and the phenotype data were fit using a multivariate linear mixed model in GEMMA (version 0.94.1) for association studies ${ }^{72}$. SNPs with missingness $>5 \%$ or a minor allele frequency $<5 \%$ were not be included in the analysis. The pipeline involves converting the SNP file into a PLINK binary PED file and generating a relatedness matrix file using default parameters. Again, the average initial weight of larvae was used as a covariate for the analysis. The statistical significance thresholds for both the Arabidopsis and Pieris GWA studies were determined using simpleM ${ }^{80}$, a multiple testing correction method for genetic association studies using correlated SNPs. To test the robustness of the GWA study results, the association tests were repeated 1000 times with randomized sample names in the phenotype file using custom scripts. $99 \%$ of the randomized datasets yielded $\leq 3$ significant SNPs at our empirical significance threshold and only one randomized dataset yielded more significant SNPs (18 compared to our empirical result of 16; Supplementary Fig. 11).

Selection scans. Nucleotide diversity $(\pi)$ and Tajima's D values were estimated using a window size of $1 \mathrm{kbp}$ and the --window-pi and --TajimaD options of the VCFtools (version 0.1.13). We analysed P. rapae SNPs across the herbivory associated genomic regions, as well as the neighbouring $100 \mathrm{kbp}$ upstream and downstream (Supplementary Figs. 4 and 5). HKA tests ${ }^{81}$ were used to compare polymorphism and divergence (compared to Pieris napi) among genes in the herbivory associated genomic regions and flanking regions. HKA tests were performed using DnaSP (version 6) ${ }^{82}$.

Arabidopsis and Pieris RNA-seq. P. rapae was raised on A. thaliana at $23^{\circ} \mathrm{C}$ and $65 \%$ relative humidity on a $16 \mathrm{~h}$ photoperiod. Samples at different time points were collected from both the plant and insect in TRIzol Reagent (Supplementary Table 4). To capture gene expression changes in response to oviposition, we compared gene expression profiles of (a) leaves with eggs (Fig. 2a,b) versus leaves without eggs $72 \mathrm{~h}$ after oviposition, and (b) eggs deposited on leaves versus eggs deposited on wax paper $72 \mathrm{~h}$ after oviposition (Fig. $2 \mathrm{c}$ ). To capture gene expression changes of the plant in response to larval feeding, we compared (c) leaves after $24 \mathrm{~h}$ of larval feeding from plants previously exposed to eggs versus leaves after $24 \mathrm{~h}$ of larval feeding from plants not previously exposed to eggs (Fig. 2d) versus control leaves never exposed to eggs or larvae. We also analysed leaves $24 \mathrm{~h}$ after mechanical wounding (Fig. 2e) to compare gene expression changes associated with larval feeding versus wounding. To identify gene expression changes in the insect associated with feeding, we compared (d) larvae after $24 \mathrm{~h}$ of feeding on leaves from plants exposed to eggs versus larvae after $24 \mathrm{~h}$ of feeding on leaves from plants not exposed to eggs versus larvae after $24 \mathrm{~h}$ of feeding on artificial diet (Fig. 2f). We also measured gene expression in butterfly pupae and adults, for comparison.

RNA was extracted using TRIzol Reagent and the RNA-seq libraries were generated using an Illumina TruSeq Stranded Total RNA Kit with Ribo-Zero Plant for Arabidopsis and Illumina TruSeq Stranded mRNA Library Prep Kit for Pieris. These libraries were sequenced on an Illumina HiSeq 2500 to generate SR50bp reads. The DNA sequence data from all three replicates of each treatment were processed with the Trimmomatic (version 0.36$)^{76} \mathrm{QC}$ pipeline, where all the bases in the reads that were below the quality score of 15 were trimmed using a sliding window of $4 \mathrm{bp}$. All reads that were less than $25 \mathrm{bp}$ in length were discarded. The trimmed SR50bp reads were aligned to their respective genomes using STAR (version 2.4.2) 83 $^{3}$ software using default parameters, except for intron minimum size, which was set to $0 \mathrm{bp}$ for both Arabidopsis and Pieris. In addition, the noncanonical junctions were removed for compatibility with Cufflinks using the RemoveNoncanonical option. After alignment, using the Cufflinks program within the Cufflinks suite (version 2.2.1) ${ }^{67}$, each library was assembled as an individual transcriptome and expression values were quantified using default parameters. The library type was set to fr-firststrand. Then all GTF files generated by Cufflinks were merged together into a single GTF using Cuffmerge. The differential expression testing between all pairs of treatments was performed using Cuffdiff tool with all the default parameters and an FDR-corrected $P$ value set to 0.001 . The differentially expressed set of genes in A. thaliana and $P$. rapae were annotated using the annotations available for their respective genomes.

Comparative transcriptome analysis. Each butterfly species was reared on its natural host plant under favourable environmental conditions in the laboratory greenhouse. $P$. rapae was raised on A. thaliana at $23^{\circ} \mathrm{C}$ and $65 \%$ relative humidity on a $16 \mathrm{~h}$ photoperiod. Colias eurytheme was raised on Medicago sativa at $26^{\circ} \mathrm{C}$ and $65 \%$ relative humidity on a $16 \mathrm{~h}$ photoperiod. Papilio polytes was raised on Citrofortunella microcarpa at $26^{\circ} \mathrm{C}$ and $65 \%$ humidity on a $16 \mathrm{~h}$ photoperiod. Heliconius cydno was raised on Passiflora oerstedii at $26^{\circ} \mathrm{C}$ and $65 \%$ relative humidity for a $13 \mathrm{~h}$ photoperiod. Samples at different time points were collected from both plants and herbivores in TRIzol Reagent. These are the same time points as those listed in Supplementary Table 4. RNA was extracted using TRIzol Reagent, and RNA-seq libraries were generated using Illumina TruSeq Stranded Total RNA Kit with Ribo-Zero Plant for the four plant species and Illumina TruSeq Stranded mRNA Library Prep Kit for the four butterfly species. These libraries were sequenced on an Illumina HiSeq 2500 to generate SR50bp reads. In addition, one library from each of the host plant species and two libraries from of each butterfly species (the egg and a larval stage) were sequenced with PE100bp in order to generate a reference transcriptome for each species. The number of reads generated from each library is listed in Supplementary Table 9.

The SR50bp and PE100bp reads were processed with the Trimmomatic QC pipeline (version 0.36$)^{76}$. All trimmed PE100bp reads and the trimmed SR50bp reads were assembled together for each host plant and herbivore system to generate a reference transcriptome with the Trinity package (version r20140717) ( $^{84}$. After excluding different isoforms, as predicted by Trinity, the number of unique coding sequences found in each plant species was: A. thaliana, 18,105; M. sativa, 43,843; C. microcarpa, 20,367; and P. oerstedii, 22,073. The number of unique coding sequences in each butterfly species was: P. rapae, 12,037; C. eurytheme, 16,277; P. polytes, 15,176; and H. cydno, 19,914. Trimmed SR50bp reads from each species were aligned to their respective reference transcriptome and differential expression analyses were performed with the Trinity package, which includes bowtie $2^{77}$ for alignment, RSEM $^{85}$ for transcript quantification and edge ${ }^{86}$ for differential expression analysis. Default parameters and an FDR-adjusted $P$ value of 0.001 were used for analyses.

We visualized the correlation among samples and treatments by generating PCA plots of the RNA-seq expression data from all four plant and butterfly species using the PtR option in the Trinity package ${ }^{84}$ (Supplementary Figs. 12 and 13). These analyses showed that the RNA-seq expression data clustered in biologically meaningful ways. For instance, we found that biological replicate samples grouped together, as did samples from similar experimental treatments (butterfly eggs versus larvae, leaf tissue exposed to eggs versus leaf tissue exposed to larvae).

For both $P$. rapae and A. thaliana, we analysed the RNA-seq data two different ways, using Cuffdiff ${ }^{67}$ and Trinity ${ }^{84}$. These different analyses produced similar numbers of differentially expressed genes, as revealed by comparing Fig. 2i,j to Fig. 4b,c. We explored this further by comparing the identity of genes across the two datasets and we found that while some differentially expressed genes were unique to one dataset or the other, many were found to be differentially expressed by both methods (Supplementary Table 10).

The differentially expressed set of genes from all four host-herbivore systems were annotated using Blast2GO (version 3.3 ) $^{87}$ using default settings. The annotated genes were manually parsed to find the common gene families. To detect true orthologues that were differentially expressed across all plants or all butterflies, we analysed the protein sequences of the differentially expressed genes with Proteinortho (version 5.11) 8,89 $^{88}$ using a Pvalue of 0.001 .

Osiris 9 paralogue detection. All Osiris 9 protein sequences from Bombyx mori, Drosophila melanogaster and Danaus plexippus were extracted from the Osiris

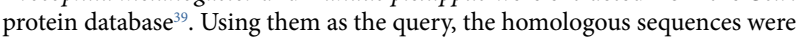
extracted from the de novo transcriptomes of all the four butterfly species and the $P$. rapae genome using tblastn similarity searches. A multiple sequence alignment of all extracted Osiris 9 protein sequences, including the Osiris 11 and Osiris 14 from B. mori, was generated using MAFFT (version $6.847 \mathrm{~b}$ ) ${ }^{90}$ with the L-INS-i algorithm. A phylogenetic tree was reconstructed using FastTree (version 2.1.10) ${ }^{91}$, which infers approximately maximum likelihood phylogenies from alignments of protein sequences. All the default options and the JTT + CAT model were used for tree inference.

Osiris $9 E$ gene spatial and temporal assay. For the temporal assay of Osiris $9 E$ expression in butterflies, whole body tissue samples were collected at ten stages of development for all four butterfly species, except for adults where wings and eyes were excluded. Multiple individuals were pooled at early instar stages $(24 \mathrm{~h}$ to $72 \mathrm{~h}$ instar) because of their small size. For the rest of the stages, a single individual was used for each RNA extraction. The developmental stages were: $24 \mathrm{~h}$ larvae, $48 \mathrm{~h}$ larvae, $72 \mathrm{~h}$ larvae, 2nd instar larva, 3rd instar larva, 4th instar larva, 5 th instar larva, pre-pupa, $72 \mathrm{~h}$ pupa and adult. For the spatial assay of Osiris $9 E$ expression in larvae, the head, foregut, midgut and skin were dissected out for 
each individual. RNA was extracted using TRIzol Reagent, and cDNA for each sample was synthesized using a High-Capacity cDNA Reverse Transcription Kit

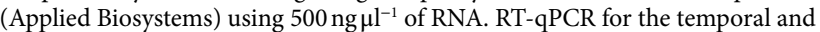
spatial samples of each species was performed using SsoAdvanced SYBR Green Supermix (Bio-Rad) and a CFX96 Optical Reaction Module thermocycler (BioRad). Elongation factor 1 alpha $(E F 1 \alpha)$ served as a normalizing gene to measure the relative expression of Osiris $9 E$ in each sample for all four butterfly systems, the primer sequences for each species are in Supplementary Table 11.

Reporting Summary. Further information on experimental design is available in the Nature Research Reporting Summary linked to this article.

Code availability. All custom codes are available from the authors upon request.

Data availability. All the DNA-seq and RNA-seq raw reads generated during this study have been deposited in the NCBI SRA database under the study accession: SRP134094. The assembled Pieris rapae genome (v1_HIRISE) is available on the Lepidopteran genome database, Lepbase, and all eight de novo transcriptome assemblies have been deposited in the DDBJ/EMBL/GenBank TSA database, under the following accession numbers: Arabidopsis thaliana (GGJX00000000), Medicago sativa (GGKA00000000), Citrus $x$ microcarpa (GGJQ00000000), Passiflora oerstedii (GGJO00000000), Pieris rapae (GGJY00000000), Colias eurytheme (GGJZ00000000), Papilio poytes (GGKB00000000) and Heliconius cydno (GGKC00000000).

Received: 23 August 2017; Accepted: 2 July 2018; Published online: 3 August 2018

\section{References}

1. Futuyma, D. J. Some current approaches to the evolution of plant-herbivore interactions. Plant Species Biol. 15, 1-9 (2000).

2. Futuyma, D. J. \& Agrawal, A. A. Macroevolution and the biological diversity of plants and herbivores. Proc. Natl Acad. Sci. USA 106, 18054-18061 (2009)

3. Ehrlich, P. R. \& Raven, P. H. Butterflies and plants: a study in coevolution. Evolution 18, 586-608 (1964).

4. Howe, G. A. \& Jander, G. Plant immunity to insect herbivores. Annu. Rev. Plant. Biol. 59, 41-66 (2008).

5. Berenbaum, M. R. Postgenomic chemical ecology: from genetic code to ecological interactions. J. Chem. Ecol. 28, 873-896 (2002)

6. Li, W., Schuler, M. A. \& Berenbaum, M. R. Diversification of furanocoumarin-metabolizing cytochrome P450 monooxygenases in two papilionids: specificity and substrate encounter rate. Proc. Natl Acad. Sci. USA 100 (Suppl 2), 14593-14598 (2003).

7. Wittstock, U. et al. Successful herbivore attack due to metabolic diversion of a plant chemical defense. Proc. Natl Acad. Sci. USA 101, 4859-4864 (2004).

8. Petschenka, G. et al. Stepwise evolution of resistance to toxic cardenolides via genetic substitutions in the $\mathrm{Na}^{+} / \mathrm{K}^{+}$-ATPase of milkweed butterflies (Lepidoptera: Danaini). Evolution 67, 2753-2761 (2013).

9. Groen, S. C. \& Whiteman, N. K. Using Drosophila to study the evolution of herbivory and diet specialization. Curr. Opin. Insect Sci. 14, 66-72 (2016).

10. Schuman, M. C. \& Baldwin, I. T. The layers of plant responses to insect herbivores. Annu. Rev. Entomol. 61, 373-394 (2016).

11. Huang, D. W., Sherman, B. T. \& Lempicki, R. A. Systematic and integrative analysis of large gene lists using DAVID bioinformatics resources. Nat. Protoc. 4, 44-57 (2009).

12. Mikkelsen, M. D., Hansen, C. H., Wittstock, U. \& Halkier, B. A. Cytochrome P450 CYP79B2 from Arabidopsis catalyzes the conversion of tryptophan to indole-3-acetaldoxime, a precursor of indole glucosinolates and indole-3acetic acid. J. Biol. Chem. 275, 33712-33717 (2000).

13. Kim, J. I., Dolan, W. L., Anderson, N. A. \& Chapple, C. Indole glucosinolate biosynthesis limits phenylpropanoid accumulation in Arabidopsis thaliana. Plant Cell 27, 1529-1546 (2015).

14. Rajniak, J., Barco, B., Clay, N. K. \& Sattely, E. S. A new cyanogenic metabolite in Arabidopsis required for inducible pathogen defence. Nature 525, 376-379 (2015).

15. Hopkins, R. J., van Dam, N. M. \& van Loon, J. J. Role of glucosinolates in insect-plant relationships and multitrophic interactions. Annu. Rev. Entomol. 54, 57-83 (2009)

16. Wheat, C. W. et al. The genetic basis of a plant-insect coevolutionary key innovation. Proc. Natl Acad. Sci. USA 104, 20427-20431 (2007)

17. Edger, P. P. et al. The butterfly plant arms-race escalated by gene and genome duplications. Proc. Natl Acad. Sci. USA 112, 8362-8366 (2015).

18. Li, J., Zhao, J., Rose, A. B., Schmidt, R. \& Last, R. L. Arabidopsis phosphoribosylanthranilate isomerase: molecular genetic analysis of triplicate tryptophan pathway genes. Plant Cell 7, 447-461 (1995).

19. Bartels, S. et al. The family of Peps and their precursors in Arabidopsis: differential expression and localization but similar induction of patterntriggered immune responses. J. Exp. Bot. 64, 5309-5321 (2013).
20. Brachi, B. et al. Coselected genes determine adaptive variation in herbivore resistance throughout the native range of Arabidopsis thaliana. Proc. Natl Acad. Sci. USA 112, 4032-4037 (2014).

21. Müller, R. et al. Differential effects of indole and aliphatic glucosinolates on lepidopteran herbivores. J. Chem. Ecol. 36, 905-913 (2010).

22. Hill, J. et al. Cryptic, extensive and non-random chromosome reorganization revealed by a butterfly chromonome. Preprint at https://www.biorxiv.org/ content/early/2018/03/02/233700 (2018).

23. Maeki, K. \& Remignton, C. Studies of the chromosomes of North American Rhopalocera. J. Lep. Soc. 14, 37-56 (1960).

24. Noctor, G., Queval, G., Mhamdi, A., Chaouch, S. \& Foyer, C. H. Glutathione Arab. Book 9, e0142 (2011).

25. Nakamura, M., Baldwin, D., Hannaford, S., Palka, J. \& Montell, C. Defective proboscis extension response (DPR), a member of the Ig superfamily required for the gustatory response to salt. J. Neurosci. 22, 3463-3472 (2002).

26. Hilker, M. \& Fatouros, N. E. Plant responses to insect egg deposition. Annu. Rev. Entomol. 60, 493-515 (2015).

27. Jones, J. D. \& Dangl, J. L. The plant immune system. Nature 444, 323-329 (2006).

28. Little, D., Gouhier-Darimont, C., Bruessow, F. \& Reymond, P. Oviposition by pierid butterflies triggers defense responses in Arabidopsis. Plant Physiol. 143, 784-800 (2007)

29. Gouhier-Darimont, C., Schmiesing, A., Bonnet, C., Lassueur, S. \& Reymond P. Signalling of Arabidopsis thaliana response to Pieris brassicae eggs shares similarities with PAMP-triggered immunity. J. Exp. Bot. 64, 665-674 (2013)

30. Schmiesing, A., Emonet, A., Gouhier-Darimont, C. \& Reymond, P. Arabidopsis MYC transcription factors are the target of hormonal salicylic acid/jasmonic acid cross talk in response to Pieris brassicae egg extract. Plant Physiol. 170, 2432-2443 (2016).

31. Zou, X. et al. Glutathione s-transferase SIGSTE1 in Spodoptera litura may be associated with feeding adaptation of host plants. Insect Biochem. Mol. Biol. 70, 32-43 (2016).

32. Yu, Q. et al. The transcriptome response of Heliconius melpomene larvae to a novel host plant. Mol. Ecol. 25, 4850-4865 (2016).

33. Schweizer, F. et al. Arabidopsis glucosinolates trigger a contrasting transcriptomic response in a generalist and a specialist herbivore. Insect Biochem. Mol. Biol. 85, 21-31 (2017).

34. Gloss, A. D. et al. Evolution in an ancient detoxification pathway is coupled with a transition to herbivory in the Drosophilidae. Mol. Biol. Evol. 31, 2441-2456 (2014).

35. Agrios, G. Plant Pathology 5th edn (Academic, Burlington, 2005).

36. Tsuda, K. \& Somssich, I. E. Transcriptional networks in plant immunity. New Phytol. 206, 932-947 (2015).

37. Roitberg, B. D. \& Isman, M. B. Insect Chemical Ecology (Springer, New York, 1992)

38. Gerardo, N. M. et al. Immunity and other defenses in pea aphids, Acyrthosiphon pisum. Genome Biol. 11, R21 (2010).

39. Shah, N., Dorer, D. R., Moriyama, E. N. \& Christensen, A. C. Evolution of a large, conserved, and syntenic gene family in insects. G3 2, 313-319 (2012).

40. Dorer, D. R., Rudnick, J. A., Moriyama, E. N. \& Christensen, A. C. A family of genes clustered at the triplo-lethal locus of Drosophila melanogaster has an unusual evolutionary history and significant synteny with Anopheles gambiae. Genetics 165, 613-621 (2003).

41. Hungate, E. A. et al. A locus in Drosophila sechellia affecting tolerance of a host plant toxin. Genetics 195, 1063-1075 (2013).

42. Andrade López, J. M. et al. Genetic basis of octanoic acid resistance in Drosophila sechellia: functional analysis of a fine-mapped region. Mol. Ecol. 26, 1148-1160 (2017).

43. Yassin, A. et al. Recurrent specialization on a toxic fruit in an island Drosophila population. Proc. Natl Acad. Sci. USA 113, 4771-4776 (2016).

44. Vanderauwera, S. et al. Genome-wide analysis of hydrogen peroxide-regulated gene expression in Arabidopsis reveals a high light-induced transcriptional cluster involved in anthocyanin biosynthesis. Plant Physiol. 139, 806-821 (2005).

45. Queval, G. \& Noctor, G. A plate reader method for the measurement of NAD, NADP, glutathione, and ascorbate in tissue extracts: application to redox profiling during Arabidopsis rosette development. Anal. Biochem. 363, 58-69 (2007).

46. Queval, G. et al. $\mathrm{H}_{2} \mathrm{O}_{2}$-activated up-regulation of glutathione in Arabidopsis involves induction of genes encoding enzymes involved in cysteine synthesis in the chloroplast. Mol. Plant 2, 344-356 (2009).

47. Chaouch, S. \& Noctor, G. Myo-inositol abolishes salicylic acid-dependent cell death and pathogen defence responses triggered by peroxisomal hydrogen peroxide. New Phytol. 188, 711-718 (2010).

48. Parisy, V. et al. Identification of PAD2 as a gamma-glutamylcysteine synthetase highlights the importance of glutathione in disease resistance of Arabidopsis. Plant J. 49, 159-172 (2007).

49. Schlaeppi, K., Bodenhausen, N., Buchala, A., Mauch, F. \& Reymond, P. The glutathione-deficient mutant pad2-1 accumulates lower amounts of glucosinolates and is more susceptible to the insect herbivore Spodoptera littoralis. Plant J. 55, 774-786 (2008). 
50. Loudet, O. et al. Natural variation for sulfate content in Arabidopsis thaliana is highly controlled by APR2. Nat. Genet. 39, 896-900 (2007).

51. Carrillo, R. A. et al. Control of synaptic connectivity by a network of Drosophila IgSF cell surface proteins. Cell 163, 1770-1782 (2015).

52. Whiteman, N. K. et al. Genes involved in the evolution of herbivory by a leaf-mining, drosophilid fly. Genome Biol. Evol. 4, 900-916 (2012).

53. Green, M. \& Sambrook, J. Molecular Cloning: A Laboratory Manual 4th edn (Cold Spring Harbor Laboratory Press, New York, 2012).

54. Putnam, N. H. et al. Chromosome-scale shotgun assembly using an in vitro method for long-range linkage. Genome Res. 26, 342-350 (2016).

55. Marçais, G. \& Kingsford, C. A fast, lock-free approach for efficient parallel counting of occurrences of k-mers. Bioinformatics 27, 764-770 (2011).

56. Leggett, R. M., Clavijo, B. J., Clissold, L., Clark, M. D. \& Caccamo, M. NextClip: an analysis and read preparation tool for Nextera Long Mate Pair libraries. Bioinformatics 30, 566-568 (2014).

57. Gnerre, S. et al. High-quality draft assemblies of mammalian genomes from massively parallel sequence data. Proc. Natl Acad. Sci. USA 108, 1513-1518 (2011).

58. Parra, G., Bradnam, K. \& Korf, I. CEGMA: a pipeline to accurately annotate core genes in eukaryotic genomes. Bioinformatics 23, 1061-1067 (2007).

59. Boetzer, M., Henkel, C. V., Jansen, H. J., Butler, D. \& Pirovano, W. Scaffolding pre-assembled contigs using SSPACE. Bioinformatics 27, 578-579 (2011).

60. Simão, F. A., Waterhouse, R. M., Ioannidis, P., Kriventseva, E. V. \& Zdobnov, E. M. BUSCO: assessing genome assembly and annotation completeness with single-copy orthologs. Bioinformatics 31, 3210-3212 (2015).

61. Kiełbasa, S. M., Wan, R., Sato, K., Horton, P. \& Frith, M. C. Adaptive seeds tame genomic sequence comparison. Genome Res. 21, 487-493 (2011).

62. Shen, J. et al. Complete genome of Pieris rapae, a resilient alien, a cabbage pest, and a source of anti-cancer proteins. F1000 Res. 5, 2631 (2016).

63. Magrane, M. \& UniProt Consortium. UniProt Knowledgebase: a hub of integrated protein data. Database 2011, bar009 (2011).

64. Meslin, C. et al. Digestive organ in the female reproductive tract borrows genes from multiple organ systems to adopt critical functions. Mol. Biol. Evol. 32, 1567-1580 (2015).

65. Trapnell, C. et al. Differential gene and transcript expression analysis of RNA-seq experiments with TopHat and Cufflinks. Nat. Protoc. 7, 562-578 (2012).

66. Kim, D. et al. TopHat2: accurate alignment of transcriptomes in the presence of insertions, deletions and gene fusions. Genome Biol. 14, R36 (2013).

67. Trapnell, C. et al. Transcript assembly and quantification by RNA-seq reveals unannotated transcripts and isoform switching during cell differentiation. Nat. Biotechnol. 28, 511-515 (2010).

68. Holt, C. \& Yandell, M. MAKER2: an annotation pipeline and genomedatabase management tool for second-generation genome projects. BMC Bioinform 12, 491 (2011)

69. Stanke, M., Diekhans, M., Baertsch, R. \& Haussler, D. Using native and syntenically mapped cDNA alignments to improve de novo gene finding. Bioinformatics 24, 637-644 (2008).

70. Tate, R., Hall, B., DeRego, T. \& Geib, S. Annie: The ANNotation Information Extractor (Version 1.0) (2014); http://genomeannotation.github.io/annie.

71. Horton, M. W. et al. Genome-wide patterns of genetic variation in worldwide Arabidopsis thaliana accessions from the RegMap panel. Nat. Genet. 44, 212-216 (2012).

72. Zhou, X. \& Stephens, M. Efficient multivariate linear mixed model algorithms for genome-wide association studies. Nat. Methods 11, 407-409 (2014).

73. Nielsen, R., Paul, J. S., Albrechtsen, A. \& Song, Y. S. Genotype and SNP calling from next-generation sequencing data. Nat. Rev. Genet. 12, 443-451 (2011).

74. Chang, C. C. et al. Second-generation PLINK: rising to the challenge of larger and richer datasets. GigaScience 4, 1-16 (2015).

75. Alexander, D. H., Novembre, J. \& Lange, K. Fast model-based estimation of ancestry in unrelated individuals. Genome Res. 19, 1655-1664 (2009).

76. Bolger, A. M., Lohse, M. \& Usadel, B. Trimmomatic: a flexible trimmer for Illumina sequence data. Bioinformatics 30, 2114-2120 (2014).

77. Langmead, B. \& Salzberg, S. L. Fast gapped-read alignment with Bowtie 2 . Nat. Methods 9, 357-359 (2012).

78. DePristo, M. A. et al. A framework for variation discovery and genotyping using next-generation DNA sequencing data. Nat. Genet. 43, 491-498 (2011).

79. Van der Auwera, G. A. et al. From FastQ data to high confidence variant calls: the Genome Analysis Toolkit best practices pipeline. Curr. Protoc. Bioinform. 43, 11.10.1-11.10.33 (2013).

80. Gao, X., Starmer, J. \& Martin, E. R. A multiple testing correction method for genetic association studies using correlated single nucleotide polymorphisms. Genet. Epidemiol. 32, 361-369 (2008).
81. Hudson, R. R., Kreitman, M. \& Aguade, M. A test of neutral molecular evolution based on nucleotide data. Genetics 116, 153-159 (1987).

82. Rozas, J. et al. DnaSP 6: DNA sequence polymorphism analysis of large datasets. Mol. Biol. Evol. 34, 3299-3302 (2017).

83. Dobin, A. et al. STAR: ultrafast universal RNA-seq aligner. Bioinformatics 29, 15-21 (2013).

84. Haas, B. J. et al. De novo transcript sequence reconstruction from RNA-seq using the Trinity platform for reference generation and analysis. Nat. Protoc. 8, 1494-1512 (2013).

85. Li, B. \& Dewey, C. N. RSEM: accurate transcript quantification from RNA-seq data with or without a reference genome. BMC Bioinform 12, 323 (2011)

86. Robinson, M. D., McCarthy, D. J. \& Smyth, G. K. edgeR: a Bioconductor package for differential expression analysis of digital gene expression data. Bioinformatics 26, 139-140 (2010).

87. Conesa, A. et al. Blast2GO: a universal tool for annotation, visualization and analysis in functional genomics research. Bioinformatics 21, 3674-3676 (2005)

88. Lechner, M. et al. Proteinortho: detection of (co-)orthologs in large-scale analysis. BMC Bioinform 12, 124 (2011).

89. Lechner, M. et al. Orthology detection combining clustering and synteny for very large datasets. PLoS ONE 9, e105015 (2014).

90. Katoh, K. \& Toh, H. Recent developments in the MAFFT multiple sequence alignment program. Brief. Bioinform. 9, 286-298 (2008).

91. Price, M. N. et al. FastTree: computing large minimum-evolution trees with profiles instead of a distance matrix. Mol. Biol. Evol. 26, 1641-1650 (2009).

92. Heikkilä, M., Kaila, L., Mutanen, M., Peña, C. \& Wahlberg, N. Cretaceous origin and repeated tertiary diversification of the redefined butterflies. Proc. R. Soc. B 279, 1093-1099 (2012).

\section{Acknowledgements}

We thank E. Westerman, R. Marquez, L. Southcott, A. Russell and G. Garcia for assistance with experiments and D. Samac for generously providing Medicago sativa seeds. N. Saleh helped with generating the inbred P. rapae material for the sequencing the initial genome assembly. We also thank C. Sahagun for assisting with the butterfly photography. This project was funded by NIH grant GM108626 and NSF grant IOS1452648 to M.R.K., funds from and University of Pittsburgh, the Pew Charitable Trust and Neubauer funds from the University of Chicago.

\section{Author contributions}

S.N. designed the project, collected and analysed data for the GWA studies, RNA-seq and comparative transcriptome studies, and co-wrote the manuscript. J.A.H. and C.W.W sequenced and assembled the P. rapae genome. K.D., C.S. and W.Z. helped collect and raise butterflies and contributed to the GWA experiments. K.D. analysed temporal and spatial expression patterns for Osi9E expression. N.I.M. generated the inbred P. rapae line used for genome sequencing, and N.I.M., C.M. and N.L.C. generated the RNA-sec data used for P. rapae genome annotation. E.S.-R. collected the North Dakota P. rapae individuals for GWA studies. J.B. provided Arabidopsis accessions for GWA studies. M.R.K. designed and directed the project, and co-wrote the manuscript. All authors contributed to editing the manuscript.

\section{Competing interests}

The authors declare no competing interests.

\section{Additional information}

Supplementary information is available for this paper at https://doi.org/10.1038/ s41559-018-0629-9.

Reprints and permissions information is available at www.nature.com/reprints. Correspondence and requests for materials should be addressed to M.R.K. Publisher's note: Springer Nature remains neutral with regard to jurisdictional claims in published maps and institutional affiliations.

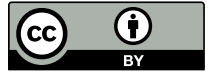

Open Access This article is licensed under a Creative Commons Attribution 4.0 International License, which permits use, sharing, adaptation, distribution and reproduction in any medium or format, as long as you give appropriate credit to the original author(s) and the source, provide a link to the Creative Commons license, and indicate if changes were made. The images or other third party material in this article are included in the article's Creative Commons license, unless indicated otherwise in a credit line to the material. If material is not included in the article's Creative Commons license and your intended use is not permitted by statutory regulation or exceeds the permitted use, you will need to obtain permission directly from the copyright holder. To view a copy of this license, visit http:// creativecommons.org/licenses/by/4.0/. 


\section{Reporting Summary}

Nature Research wishes to improve the reproducibility of the work that we publish. This form provides structure for consistency and transparency in reporting. For further information on Nature Research policies, see Authors \& Referees and the Editorial Policy Checklist.

\section{Statistical parameters}

When statistical analyses are reported, confirm that the following items are present in the relevant location (e.g. figure legend, table legend, main text, or Methods section).

n/a $\mid$ Confirmed

$\square \bigotimes$ The exact sample size $(n)$ for each experimental group/condition, given as a discrete number and unit of measurement

$\square$ \ An indication of whether measurements were taken from distinct samples or whether the same sample was measured repeatedly

$\square$ The statistical test(s) used AND whether they are one- or two-sided

Only common tests should be described solely by name; describe more complex techniques in the Methods section.

$\square$ \ A description of all covariates tested

$\square$ \A description of any assumptions or corrections, such as tests of normality and adjustment for multiple comparisons

$\square$ A full description of the statistics including central tendency (e.g. means) or other basic estimates (e.g. regression coefficient) AND

$\triangle$ variation (e.g. standard deviation) or associated estimates of uncertainty (e.g. confidence intervals)

$\square$ For null hypothesis testing, the test statistic (e.g. $F, t, r$ ) with confidence intervals, effect sizes, degrees of freedom and $P$ value noted

Give $P$ values as exact values whenever suitable.

Х $\square$ For Bayesian analysis, information on the choice of priors and Markov chain Monte Carlo settings

Х $\square$ For hierarchical and complex designs, identification of the appropriate level for tests and full reporting of outcomes

\ $\square$ Estimates of effect sizes (e.g. Cohen's $d$, Pearson's $r$ ), indicating how they were calculated

$\varnothing$ Clearly defined error bars

State explicitly what error bars represent (e.g. SD, SE, CI)

Our web collection on statistics for biologists may be useful.

\section{Software and code}

Policy information about availability of computer code

Data collection No software was used

Data analysis Jellyfish v2.1.3,Nextclip (version 0.8), bbduk v34.94,AllpathsLG (version 50960),CEGMA (version 2.5),SSPACE v2,BUSCO v3,LAST (version 714), tophat2 (version 2.0.9), cufflinks package (version 2.2.1), RepeatMasker package (4.0.3) ,RepeatModeler package (1.0.8), Maker package (version 2.31-6),Augustus gene finder (version 2.7),Annie: the ANNotation Information Extractor (Version 1.0), GEMMA (version 0.94.1), Trimmotatic (version 0.36),PICARD tools (version 1.141), GATK (version 3.4), simpleM,STAR (version 2.4.2), Cuffdiff pipeline (version 2.2.1), Trinity package (version r20140717),Blast2GO (version 3.3), Proteinortho (version 5.11), MAFFT v6.847b,FastTree (version 2.1.10), VCFtools (version 0.1.13), PLINK (version 2.0),ADMIXTURE (version 1.3)

For manuscripts utilizing custom algorithms or software that are central to the research but not yet described in published literature, software must be made available to editors/reviewers upon request. We strongly encourage code deposition in a community repository (e.g. GitHub). See the Nature Research guidelines for submitting code \& software for further information. 
Policy information about availability of data

All manuscripts must include a data availability statement. This statement should provide the following information, where applicable:

- Accession codes, unique identifiers, or web links for publicly available datasets

- A list of figures that have associated raw data

- A description of any restrictions on data availability

All the DNA-seq and RNA-seq raw reads generated during this study have been deposited in the NCBI SRA database under the study accession: SRP134094. The assembled Pieris rapae genome (V1_HIRISE) is available on the Lepidopteran genome database, Lepbase, and all eight de novo transcriptome assemblies have been deposited in the DDBJ/EMBL/GenBank TSA database, under the following accession numbers: Arabidopsis thaliana (GGJX000000000), Medicago sativa (GGKA00000000), Citrus x microcarpa (GGJQ00000000), Passiflora oerstedii (GGJO00000000), Pieris rapae (GGJY00000000), Colias eurytheme (GGJZ00000000), Papilio poytes (GGKB00000000) and Heliconius cydno (GGKC00000000).

\section{Field-specific reporting}

Please select the best fit for your research. If you are not sure, read the appropriate sections before making your selection.

Life sciences

Behavioural \& social sciences

Ecological, evolutionary \& environmental sciences

For a reference copy of the document with all sections, see nature.com/authors/policies/ReportingSummary-flat.pdf

\section{Ecological, evolutionary \& environmental sciences study design}

All studies must disclose on these points even when the disclosure is negative.

Study description

1) Parallel genome-wide association studies in the Cabbage White butterfly, Pieris rapae, and its host-plant, Arabidopsis thaliana, pinpointed a small number of butterfly and plant genes that influenced herbivory: a) Host GWAS -Three replicates of each of the 96 accessions of Arabidopsis thaliana that are listed in Supplementary Table 1 were grown until they were almost ready to bolt. After taking a picture of each plant, two 5 day old, lab-grown P. rapae larvae were weighed and placed on each plant and then the plant was enclosed in a plastic sleeve bag. After $72 \mathrm{~h}$, the larvae were weighed and a new picture of the plant was taken in order to record plant surface area eaten by the larvae. The weight gained by the larvae and the total surface area eaten were calculated and used as the phenotype data for GWAS. b) Herbivore GWAS - 96 P. rapae females were collected from various locations across the US Midwest during and raised in the lab green house. Eggs were collected from each female and two 5 day old larvae from each family were weighed and placed together on a Col-0 A. thaliana plant and this was performed in triplicate. Leaf area eaten and weight gain phenotypes were assayed as described for the Host GWAS.

2) Comparative transcriptome analyses, including diverse butterfly/plant systems, showed a variety of genome-wide responses to herbivory, yet a core set of highly conserved genes in butterflies as well as their host-plants: Each butterfly species was reared on its natural host-plant under favorable environmental conditions in the laboratory greenhouse.Samples at different time points were collected from both plants and herbivores in TRIzol Reagent. These are the same time points as those listed in Supplementary Table 4.

Research sample

Comparative transcriptome analyses: Pieris rapae was raised on Arabidopsis thaliana at $23^{\circ} \mathrm{C}$ and $65 \%$ relative humidity on a 16 hour photoperiod. Colias eurytheme was raised on Medicago sativa at $26^{\circ} \mathrm{C}$ and $65 \%$ relative humidity on a 16 hour photoperiod. Papilio polytes was raised on Citrofortunella microcarpa at $26^{\circ} \mathrm{C}$ and $65 \%$ humidity on a 16 hour photoperiod. Heliconius cydno was raised on Passiflora oerstedii at $26^{\circ} \mathrm{C}$ and $65 \%$ relative humidity for a 13 hour photoperiod.

Sampling strategy

The sample size of $96 \mathrm{~A}$. thaliana accessions and 96 P. rapae population lines was chosen for GWAS as similar studies had successfully detected the underlying genes using similar or even low number of samples (Atwell, S. et al. Nature 465, 627-631 (2010); Karasov, Talia L. et al. Nature 512.7515 (2014): 436-440.

Data collection

GWAS data: a) Host GWAS -After taking a picture of each plant, two 5 day old, lab-grown P. rapae larvae were weighed and placed on each plant and then the plant was enclosed in a plastic sleeve bag. After $72 \mathrm{~h}$, the larvae were weighed and a new picture of the plant was taken in order to record plant surface area eaten by the larvae. The weight gained by the larvae and the total surface area eaten were calculated and used as the phenotype data for GWAS. b) Two 5 day old larvae from each family were weighed and placed together on a Col-0 A. thaliana plant and this was performed in triplicate. Leaf area eaten and weight gain phenotypes were assayed as described for the Host GWAS. SN, KD, CS and WZ collected the data.

Comparative transcriptome analyses: Samples at different time points (Supplementary Table 4) were collected from both plants and herbivores in TRIzol Reagent by SN.

Timing and spatial scale GWAS data collection timing: April - August 2014, the P. rapae was collected during June -July 2014 because it is available abundantly in the Midwest in early summer.

Comparative transcriptome: Samples at different time points were collected from the four species of both plants and herbivores from August-October 2014

Data exclusions No data was excluded

Reproducibility All attempts at replication were successful. 
Blinding

All the phenotype measurements for GWAS were collected blindly. The investigators measured the plants and larvae randomly without knowing the name of the accession or the population line.

Did the study involve field work? $\quad$ Yes $\square$ No

\section{Field work, collection and transport}

Field conditions

Location

Access and import/export

Disturbance
96 P. rapae females were collected from various locations across the US Midwest during June-July 2014 (Summer).

These butterflies included 57 from around the University of Chicago campus, 19 from Schaumburg, IL, and 16 from North Dakota, 3 from downtown Chicago, and 1 from Carolina Biological Supply.

No permits required

No disturbance

\section{Reporting for specific materials, systems and methods}

\section{Materials \& experimental systems}

n/a Involved in the study

$\searrow \square$ Unique biological materials

Х $\square$ Antibodies

Х $\square$ Eukaryotic cell lines

Х $\square$ Palaeontology

$\square$ \ Animals and other organisms

$\searrow \mid \square$ Human research participants

\author{
Methods \\ $\mathrm{n} / \mathrm{a}$ Involved in the study \\ X $\square$ ChIP-seq \\ Х $\square$ Flow cytometry \\ Х $\square$ MRI-based neuroimaging
}

\section{Animals and other organisms}

Policy information about studies involving animals; ARRIVE guidelines recommended for reporting animal research

Laboratory animals

Wild animals

Field-collected samples
For genome assembly, Pieris rapae were sampled in September 2013 from a continuous, inbred lab colony established in October 2012 from 4 females collected in Rochester, Pennsylvania.

The study did not involve wild animals.

The growth chamber conditions for growing field collected Pieris rapae were $23^{\circ} \mathrm{C}$ day $/ 210 \mathrm{C}$ night and $60 \%$ relative humidity on a 16 hour photoperiod. 